\title{
Understanding Interactions Histological, Cyto- logical, and Molecular Among the fungus P. Striiformis Tritici and Wheat
}

\section{Entendiendo las Interacciones Histológicas, Citológicas, y Moleculares Entre El Hongo P. Striiformis Tritici y el Trigo}

I International Congress of

Science and Technology Morona Santiago-CICTMS 2020

Corresponding Author:

Valeria Moreno Heredia valeria.morenoheredia@ uqconnect.edu.au

Published: 29 August 2021

Production and Hosting by Knowledge E

(c) Valeria Moreno Heredia. This article is distributed under the terms of the Creative Commons Attribution License, which permits unrestricted use and redistribution provided that the original author and source are credited.

\section{Valeria Moreno Heredia}

Investigadora Independiente, Riobamba, Ecuador

\section{Abstract}

Yellow rust is caused by the fungus Puccinia striiformis f.sp.tritici (Pst), which due to its great migratory capacity, adaptation to different environments, and high levels of mutation; is one of the most devastating wheat diseases worldwide. Due to this, several strategies have been implemented to control the disease, the best being genetic improvement. The key to develop resistant cultivars is understanding the interactions between wheat and Pst. Therefore, this work synthesizes the most important investigations carried out in the last 30 years regarding: cellular, histological, and molecular interactions between wheat and Pst. This will allow a deeper and more complete understanding of the interaction between resistance and virulence genes in the yellow rust disease. The results of this work revealed that the early stage of infection, in susceptible and resistant cultivars, is the same qualitatively, but not quantitatively. However, a clear difference at the histological and molecular level, in terms of the amount and type of genes expressed, begins 48 hours after infection. It was also found that the haustorium, in addition to absorbing nutrients from the host; can also manipulate its metabolism to benefit itself, and can make some nutrients on its own.

Keywords: haustorio, Puccinia striiformis f.sp.tritici, histological, resistance genes, virulence genes.

\section{Resumen}

La roya amarilla es causada por el hongo Puccinia striiformis f.sp.tritici (Pst), el cual debido a su gran capacidad migratoria, adaptación a diferentes ambientes, y niveles altos de mutación; es la enfermedad más devastadoras del trigo a nivel mundial. Debido a esto, varias estrategias han sido implementadas para controlar la enfermedad, siendo la mejor, el mejoramiento genético. La clave para desarrollar cultivares resistentes, es el entendimiento de las interacciones entre el trigo y Pst. Por lo tanto, este trabajo sintetiza las investigaciones más importantes realizadas en los últimos 30 años, en cuanto a interacciones celulares, histológicas y moleculares entre el trigo y Pst. Esto permitirá un entendimiento más profundo y completo de la interacción entre los genes de resistencia y virulencia, en la enfermedad de la roya. Los resultados revelaron que la fase temprana de infección en cultivares susceptibles y resistentes, es igual cualitativamente, pero no cuantitativamente. Sin embargo, una diferencia clara a nivel histológico y molecular, en cuanto a la cantidad y al tipo de genes expresados, empieza 48 hr post infección. También, se halló que el haustorio además de absorber nutrientes del huésped, también manipula el metabolismo de éste para su beneficio y puede elaborar algunos nutrientes por sí mismo.

Palabras Clave: haustorio, Puccinia striiformis f.sp.tritici, histológico, genes de resistencia, genes de virulencia 


\section{Introducción}

Los cereales contribuyen el $50 \%$ de las calorías diarias necesarias para la población mundial [1]. El trigo en particular, es el cereal más cultivado en todo el mundo. Ocupa un área total de 219 millones de ha/año, es decir 42 y 57 millones de ha/año más que el maíz y el arroz, respectivamente [2]. En 2019 la producción global de trigo fue de 763 millones de toneladas, un 4.2\% más que en 2018 y se estima que la producción tendrá que duplicarse para el año 2050, dado el aumento de la población a 10 billones y un posible crecimiento económico [1, 3]. Actualmente, este crecimiento se ve amenazado por el calentamiento global, reducción de agua, disminución de tierra arable y un aumento en pestes. Por ello, es importante aumentar el rendimiento del grano por unidad de área de tierra [1]. Una herramienta para lograr este objetivo es el control de enfermedades. Anualmente se obtiene cerca de USD \$50 billones por la comercialización del trigo, pero enfermedades como la roya amarilla causan pérdidas de 5.47 millones de toneladas de trigo al año, equivalentes a USD \$979 millones de dólares [4,5]. Esto la convierte en la enfermedad del trigo más importante en términos económicos. El agente causal de la roya amarilla o estriada en el trigo, es el hongo Puccinia striiformis f.sp.tritici (Pts). Este posee una gran capacidad de dispersión, adaptación a diferentes ambientes, altas tasas de mutación de razas y diversidad genética [6]. Lo que ha hecho posible la distribución mundial de Pst, causando pérdidas importantes en varios continentes. Actualmente la estrategia más económica y amigable con el medio ambiente, es el control genético [7]. El control genético crea plantas resistentes, a una o varias razas del hongo Pst. Sin embargo, mantener estas nuevas razas resistentes a nivel regional y continental requiere un mejor entendimiento de las interacciones huésped-patógeno [8]. Esto sin mencionar que el hongo Puccinia striiformis, en general, posee el ciclo de vida más complejo dentro de todos los hongos patógenos de las plantas. Pst al ser un biotrofo obligado, no puede ser cultivado sintéticamente. Por lo tanto, el estudio de los cambios histológicos, citológicos y moleculares tanto en Pst como en la planta durante el proceso de infección, es complicado [9]. En vista de esto, el objetivo de este artículo es recopilar investigaciones de los últimos treinta años en cuanto a interacciones histológicas, citológicas, y moleculares entre el trigo y Pst. Este estudio beneficiará a investigadores, profesores, agricultores, estudiantes y personas interesadas en mantener la seguridad alimentaria del trigo, dada su importancia nutricional, económica y científica a nivel mundial.

\section{Metodología}

En este artículo se realizó una búsqueda sistemática de artículos científicos, reportes, y libros realizados en los últimos 30 años, por universidades y asociaciones profesionales. Además, se buscó que las fuentes de investigación sean de carácter nacional e internacional. A nivel internacional se investigó en las revistas: Nature, Science, APS, Frontiers, Plos; mientras que a nivel regional en las revistas: Scielo, Redalyc y Latindex. También se utilizaron bases de datos y buscadores prestigiosos a escala global como: Elsevier, NCBI, Springer, Pubmed, Librearía Wiley, y Google Académico. 


\section{Desarrollo y Discusión}

\subsection{Interacciones histológicas y citológicas}

\subsubsection{Interacciones compatibles e incompati- bles entre el trigo y Puc- cinia striiformis f.sp.tritici}

Existen dos tipos de interacciones trigoPst: incompatibles y compatibles. En la interacción incompatible, el producto de un gen de resistencia $(R)$ de la planta, reconoce un producto de un gen de avirulencia (avr) del patógeno, y como resultado la planta desencadena una respuesta hipersensible $(\mathrm{RH})$ que termina en muerte celular [10]. En las interacciones compatibles, la planta carece de genes de resistencia y por lo tanto su defensa es menos efectiva. La resistencia desplegada por cultivares resistentes puede ser cualitativa (resistencia vertical) o cuantitativa (resistencia horizontal) [7].

\subsubsection{Resistencia cualitativa}

Un ejemplo de esta resistencia fue encontrado en el cultivar resistente Suwon 11, el cual desplegó una respuesta hipersensitiva cuando se lo puso en contacto con el patógeno CY23 [11]. Esta reacción tuvo efecto 18 hr después de la infección, y a partir de 72 hr post infección (hpi) casi todos los sitios de infección poseían células del huésped con necrosis. Dos días después, un gran número de células huésped murieron, pero la propagación del hongo fue detenida [11]. Algunos estudios histológicos y citológicos han revelado que las plantas de trigo resistentes secretan más lignina, quitinasa, tionina y glicoproteínas ricas en hidroxiprolina, que aquellas susceptibles [12-14]. Igualmente sucede con las especies reactivas de oxígeno (ROS): $\mathrm{H}_{2} \mathrm{O}_{2}$ y $\mathrm{O}^{-}$. Estas se encuentran en mayor medida en cultivares resistentes hasta 96 hpi [13]. Sin embargo, se encontró que, en la fase temprana de infección, las interacciones compatibles e incompatibles, muestran un crecimiento similar del hongo. No es hasta 48 hpi, que la inhibición del crecimiento del hongo es significativamente mayor en la interacción incompatible (cultivares resistentes). En las plantas resistentes, el citoplasma de la hifa se llena de sacos y parches dispersos de electrones. El cuerpo haustorio empieza a rodearse de material denso en electrones, mientras que el citoplasma de las células madre haustorio es vacuolado. Los orgánulos del citoplasma se agregan y se desintegran; mientras que la capa alrededor del cuerpo haustorio se ensancha de forma irregular, acumulando depósitos granulares. Finalmente, esta membrana se arruga y se vuelve necrótica $[12,14]$.

\subsubsection{Resistencia cuantitativa}

Esta resistencia también conocida como resistencia de plantas adultas (RPA), puede desencadenar una respuesta de hipersensibilidad. Sin embrago, en términos generales utiliza estrategias más sutiles que la muerte celular. Por ejemplo, los genes de resistencia Yr18 y Yr29 no están asociados con una respuesta hipersensible (RH) 
significativa. Pero, el cultivar Xingzi 9104 posee un gen de resistencia de RPA que es asociado con una RH continua y una producción localizada de ROS; además de un retardo en la formación de haustorio e inhibición considerable en la formación de hifas secundarias. Esto resulta en una disminución de microcolonias en plantas adultas [15]. La resistencia cuantitativa también es conocida como resistencia de plantas adultas a temperaturas altas (HTAP). Ésta es usualmente nula o baja en plantas jóvenes, pero existen excepciones. El gen Yr36 se expresa en plantas jóvenes y adultas, pero es activado solamente a temperaturas altas $\left(33^{\circ} \mathrm{C}\right.$ en el día y $12^{\circ} \mathrm{C}$ la noche). Al estudiar el gen Yr36 con el patotipo CYR29, las observaciones histológicas mostraron que las plantas con el gen Yr36 podían detener el crecimiento de hifas secundarias, las células del huésped mostraban necrosis y muerte celular, y las especies oxidativas reactivas se acumulaban a niveles importantes una vez que las hifas empezaron a extenderse [16]. Otro estudio comparó las respuestas del cultivar Avocet $S$ (susceptible) con el cultivar Kariega (resistencia completa de planta adulta), luego de una infección con el patotipo $6 E 22$ [17]. Cuatro días después de la inoculación, los primeros sitios necróticos fueron observados en Kariega, pero no en Avocet S. Cinco días post infección, las células mesófilas en Kariega mostraron lignificación, mientras que las células de Avocet $\mathrm{S}$, no (Refiérase a la Figura 1). Seis días después de la infección, el cultivar susceptible finalmente mostro células necróticas. El octavo día, el número de colonias en Avocet $\mathrm{S}$ mostró un crecimiento descomunal, tanto que no se pudo cuantificar el número de esporas en la hoja bandera.

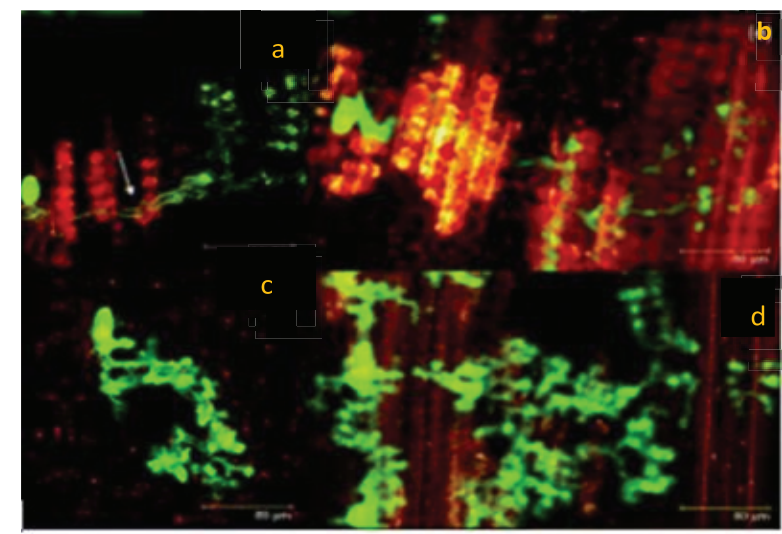

\section{Figure 1}

El hongo fue coloreado con Uvitex y analizado con microscopia de escaneo laser confocal. La vecinidad inmediata al hongo esta mostrada en verde. Tejido necrótico apareció 5 dpi en Kariega, mostrado en rojo amarillento (a), pero no en Avocet S (c). Las células necróticas rodearon al hongo completamente 7 dpi en Kariega (b), mientras que Avocet S presento pocas células necróticas dispersas (d) (Modificado de Moldenhauer et al., 2006). 


\subsection{Interacciones moleculares}

\subsubsection{Genes up-regulados en el trigo durante interacciones compatibles e incompati- bles trigo-Pst}

El uso de la mayoría de técnicas moleculares y genéticas para estudiar las interacciones trigo-Pst son limitadas. Esto debido a la difícil tarea de transformar el trigo por su complejo genoma hexaploide y a la imposible tarea de cultivar a Pst en un medio sintético. Sin embargo, algunas técnicas han logrado elucidar las funciones de ciertos genes involucrados en la interacción trigo-Pst, basándose en estudios transcriptómicos. Un estudio utilizó la tecnología ADNc-AFLP, para detectar genes diferencialmente expresados durante la interacción incompatible trigo-Pst, y la comparó con su estudio previo donde investigó las interacciones compatibles [11]. En la interacción incompatible se identificaron 1787 tránscritos up-regulados y 650 down-regulados, y se obtuvieron 255 secuencias confiables, de los cuales 113 tenían funciones putativas identificadas. El $7.4 \%$ [18] fueron del hongo Pst, 129 del trigo, y los restantes no tenían un origen claro. En la interacción compatible 966 tránscritos fueron up-regulados y 1340 down-regulados, y se identificaron 186 fragmentos de tránscritos confiables, 32 (17\%) provenientes de Pst y 87 (47\%) del trigo [19]. Ya que la interacción compatible muestra más genes desactivados en el huésped, que la interacción incompatible, podemos sugerir que el patógeno tiene la capacidad de suprimir las defensas del huésped. Debido a esto, dos veces más genes fueron desactivados en las plantas susceptibles que en las resistentes. En la interacción incompatible, 45 de los 129 genes identificados del trigo poseían alta homología compartida con genes de metabolismo y fotosíntesis, 18 con genes de defensa de enfermedades; 17 en la transducción de señales, y los restantes 33 eran genes implicados en transcripción, procesos de transporte, metabolismo de proteínas y estructura celular [11]. Mientras que en la interacción compatible se detectaron genes implicados en la energía, transducción de señales, defensa de enfermedades, y metabolismo [19].

La falta de expresión de genes de estructura celular, procesos de transporte y transcripción, en la interacción compatible; indicarían que los genes de resistencia, son los encargados de codificar estos tránscritos. Interesantemente, 161 de los 255 genes identificados en i. incompatibles, también fueron inducidos en i. compatibles; mientras que 94 fueron expresados específicamente en i. incompatibles [11]. Por lo cual, el autor determinó que 161 tránscritos, corresponden a la defensa basal de la planta. También, encontraron que los genes implicados en la transducción de señales, y defensa de enfermedades alcanzaron su punto máximo de expresión de 12 a 24 hpi, en interacciones compatibles e incompatibles [11, 19]. Mientras que los genes de resistencia fueron altamente expresados de 48 a $72 \mathrm{hpi}$, pero vuelven a niveles basales $120 \mathrm{hpi}$ [11]. Esto sugiere que la respuesta a un ataque de Pst, en la etapa inicial, es similar en interacciones compatibles e incompatibles el primer día, pero empiezan a diferir dos días después y vuelven a ser similares el quinto día de la interacción. 


\subsubsection{Perspectiva molecular de la resistencia cualitativa}

Generalmente, esta resistencia confiere inmunidad a un solo patotipo de Pst, y se presenta en todas las etapas de crecimiento de la planta. Esta es gobernada por genes mayores, que en su mayoría pueden adaptarse a Pst en unos pocos años [18].

Las líneas casi isogénicas Yr1 y Yr10 fueron estudiadas con la técnica DDRTPCR, para identificar los genes que se expresaban en diferentes tiempos, a partir de una infección de Pst. Treinta y tres de los genes encontrados fueron analizados más a fondo con qRT-PCR, y 14 produjeron niveles reproducibles de inducción o represión. Entre estos se encontraron una enzima conjugadora de ubiquitina y una proteína tipo ciclofilina. Estos genes podrían estar involucrados en la ubiquitinilacion, muerte celular programada, actividades anti fúngicas, respuestas a resistencia de enfermedades, y respuestas relacionadas con la patogénesis. Adicionalmente, se encontró que los genes a cargo de la degradación de proteínas, mediada por ubiquitina, se regulan en el trigo como respuesta a una infección de un patógeno a virulento de la roya amarilla [20]. En otro estudio se identificaron los tránscritos asociados a Yr5. Mediante un GeneChip, se encontró que la presencia de Yr5, activa tránscritos de defensa y vías de señalización comunes en una respuesta mediada por los genes $\mathrm{R}$ [21]. Esta respuesta incluye la señalización de la proteína kinasa y la producción de especies reactivas de oxigeno que conducen a una $\mathrm{RH}$.

\subsubsection{Perspectiva molecular de la resistencia cuantitativa}

La resistencia cualitativa al estar gobernada por un conjunto de genes menores, puede resistir varios años [22]. Además, a diferencia de los genes de resistencia vertical quienes únicamente actúan mediante una respuesta hypersensitiva $(R H)$; los genes de resistencia horizontal desencadenan otras respuestas, además de la $(\mathrm{RH})$.

En otro estudio se demostró que

Xingzi9104 poseía genes involucrados en ROS, biosíntesis de fenilpropanoide, fotosíntesis y metabolismo de tiamina; los cuales participan en la regulación de la lignificación y formación de azúcar [23]. En un estudio diferente, se comparó diferentes genes (Yr18, Yr29, y Yr39) de resistencia HTAP o APR [24]. Se encontró que todos los genes, expresaron un tránscrito en común, el cual era una proteína de la capa de no-clatrina, cuya función putativa es la de transporte de sustancias anti-fúngicas a través de la membrana celular para hacer contacto directo con el haustorio o hifa.

\subsubsection{Estrategias de patogenicidad de Pst en el trigo}

El haustorio es un sitio concertado de interacción entre el huésped y el patógeno, por lo que descubrir sus funciones es esencial para entender los mecanismos de supervivencia de Pst dentro del huésped.

El haustorio es ampliamente reconocido por su función en la absorción de nutrientes, pero estudios recientes han demostrado que el haustorio no solamente toma nutrientes del huésped, sino que manipula al huésped para su beneficio. Por ejemplo: TaAMT2; 3a, 
es un transportador de amonio tipo AMT2 del trigo, pero se demostró que al silenciar el gen TaAMT2; 3a, el número y longitud de hifas, así como las células madre haustorio, decreció. Sin embrago, en las plantas donde TaAMT2; 3a, no fue silenciado, Pst indujo a este transportador, para obtener nitrógeno. También se ha demostrado que Pst puede secretar efectores que son transportados fuera de la membrana extrahaustorial [25]. La proteína Pst_12806 es translocada del haustorio a los cloroplastos, donde interactúa con el C-terminal del dominio Rieske de la proteína TalSP del trigo. Esta interacción resulta en una reducción de la velocidad de transporte de electrones, reducción de fotosíntesis, y la producción de ROS derivada de cloroplastos [26]. No solo esto, se ha encontrado que Pst puede producir ciertos nutrientes por sí mismo a través de la síntesis de novo. Los genes asociados con la vía de biosíntesis de tiamina, son masivamente upregulados en Pst durante la infección. El pirofosfato de tiamina (Vitamina B1), actúa como cofactor en varias enzimas del metabolismo central del carbono: alfa-cetoglutarato deshidrogenasa $(\alpha-K G D H)$ en el ciclo de Krebs; transcetolasa en el PPS no oxidativo; y piruvato deshidrogenasa (PDH) que conecta el glucólisis y el ciclo de Krebs [24].

\section{Conclusiones}

Las interacciones trigo-Pst compatibles e incompatibles, en la fase temprana de infección (12-24 hpi), son similares en cuanto a los componentes que intervienen, pero difieren en la cantidad de los mismos. Sin embargo, la diferencia a nivel histológico y molecular se hace evidente $48 \mathrm{hr}$ post infección. Y, regresan a niveles similares 72 hpi. También, contrario al conocimiento común; algunas moléculas y efectores tanto del huésped como del parásito pueden atravesar la membrana extrahaustorial, ya sea para atacar o defenderse.

Igualmente, se encontró que ciertos genes de resistencia cuantitativa también pueden expresar una respuesta hipersensitiva $(\mathrm{RH})$, tal como lo hacen los genes de resistencia cualitativa, pero en menor medida. Además, se halló que el haustorio no solamente actúa como una herramienta pasiva de absorción de nutrientes, sino que puede manipular el metabolismo del huésped para su beneficio, y puede proveer nutrientes esenciales para sí mismo.

\section{Recomendaciones}

Se recomienda investigar más a fondo los genes que causan que las interacciones compatibles e incompatibles difieran a partir del segundo día. Determinarlo podría ser ventajoso, ya que se podría acelerar el tiempo de reacción, y el hongo no tendría oportunidad de desarrollarse. Además, se recomienda estudiar otros mecanismos mediante los cuales el hongo pueda burlar y manipular las defensas de la planta. Esto implicaría estudiar grandes poblaciones de genes de resistencia, así como de diversas razas del hongo Pst. Los estudios poblacionales, no solo a nivel regional sino global, favorecerían el control de la enfermedad. 


\section{References}

[1] Singer SD, Foroud NA, Laurie JD. Molecular improvement of grain: Target traits for a changing world. Encyclopedia of Food Security and Sustainability. 2019. Available from: https://www.sciencedirect.com/ topics/agricultural-and-biological- sciences/cereal-crop

[2] Abbate PE, Cardos MJ, Campaña LE. Manual de cultivo de trigo. $1^{\text {st }}$ ed. Argentina: International Plant Nutrition Institute; 2017. Available from: https://www.researchgate.net/publication/320465244_ El_trigo_su_difusion_importancia_como_alimento_y_consumo

[3] FAO. Estados Unidos. FAO. 2020 June 2. Available from: http://www.fao.org/worldfoodsituation/csdb/ es/.

[4] CGIAR. Mexico. Wheat.org. 2017. Available from: https://wheat.org/wheatin-the-world/.

[5] Solh M, Nazari K, Tadesse W, Wellings CR. The growing threat of stripe rust worldwide. Presented at: Proceedings of the GBR1 2012 Technical Workshop; 2012 Sep 1-4; Beijing, China.

[6] Carmona M, Sautua F. Roya amarilla del trigo: Nuevas razas en el mundo, monitoreo y uso de fungicidas. Argentina: FAUBA; 2016. Available from: http://herbariofitopatologia.agro.uba.ar/wpcontent/uploads/ 2016/03/CARMONASAUTUA_Royaamarilla2017_FAUBA.pdf

[7] Buerstmayr M, Matiasch L, Mascher F et al. Mapping of quantitative adult plant field resistance to leaf rust and stripe rust in two European winter wheat populations reveals colocation of three QTL conferring resistance to both rust pathogens. Theoretical and Applied Genetics. 2014;127:2011-2028.

[8] Sharma-Poudyal D, Chen XM, Wang A et al. Virulence characterization of international collections of the wheat stripe rust pathogen, Puccinia striiformis f.sp. tritici. The American Phytopathological Society. 2013;97:379-386.

[9] Kang Z, Tang C, Zhao J. Wheat Puccinia striiformis Interactions. Chen X, Kang Z, editors. Stripe Rust Washington, USA: SpringerScience+Business Media B.V; 2017. p. 197-198.

[10] Flor HH. The complementary gene systems in flax and flax rust. Advances in Genetics. 1956;8:29-54

[11] Wang X, Liu W, Chen X, et al. Differential gene expression in incompatible interaction between wheat and stripe rust fungus revealed by cDNA-AFLP and comparison to compatible interaction. BMC Plant Biol. 2010;10:9.

[12] Kang Z, Wang Y, Huang L. Histology and ultrastructure of incompatible combination between Puccinia striifromis and wheat cultivars with resistance of low reaction type. Sci Agric Sin. 2003;36:1026-31.

[13] Wang C, Huang L, Buchenauer $\mathrm{H}$ et al. Histochemical studies on the accumulation of reactive oxygen species (O2- and $\mathrm{H}_{2} \mathrm{O} 2$ ) in the incompatible and compatible interaction of wheat-Puccinia striiformis f. sp. tritici. Physiol Mol PlantPathol. 2007;71:230-9.

[14] Zhang H, Han Q, Wang $C$ et al. Histology and ultrastructure of resistant mechanism of a new wheat material Yilipu to Puccinia striiformis. Acta Phys Sin. 2008;38:153-64.

[15] Zhang H, Wang C, Chen $\mathrm{Y}$ et al. Histological and cytological characterization of adult plant resistance to wheat stripe rust. Plant Cell Rep. 2012;31:2121-37.

[16] Moldenhauer B, Moerschbacher A, Van Der Westhuizen A. Histological investigation of stripe rust (Puccinia striiformis f.sp. tritici) development in resistant and susceptible wheat cultivars. Plant Pathology. 2006;55:469-474.

[17] Li H, Ren B, Kang ZS, Huang LL. Comparison of cell death and accumulation of reactive oxygen species in wheat lines with or without Yr36 responding to Puccinia striiformis $\mathrm{f}$. sp. tritici under low and high temperatures at seedling and adult-plant stages. Protoplasma. 2016;253:787-802.

[18] Wang X, Tang Ch, Zhang G et al. cDNA-AFLP analysis reveals differential gene expression in compatible interaction of wheat challenged with Puccinia striiformis f. sp.tritici. BMC Genomics. 2009;10(289).

[19] Priyamvada A, Saharan MS, Tiwari R. Durable resistance in wheat. Journal of Genetics and Molecular Biology. 2011;3:108-114.

[20] Bozkurt O, Unver T, Akkaya MS. Genes associated with resistance to wheat yellow rust disease identified by differential display analysis. Physiol Mol Plant Pathol. 2008;71:251-9.

[21] Coram T, Wang M, Chen X. Transcriptome analysis of the wheat Puccinia striiformis $\mathrm{f}$. sp. Tritici interaction. Mol Plant Pathol. 2008;9:157-169.

[22] Parlevliet JE. Resistance of the nonrace-specific type. Roelfs AP, Bushnell WR, editors. The cereal rusts: Diseases, distribution, epidemiology, and control. New York, USA: Academic Press;1985. p. 501-525.

[23] Hao $\mathrm{Y}$, Wang T, Wang $\mathrm{K}$ et al. Transcriptome analysis provides insights into the mechanisms underlying wheat plant resistance to stripe rust at the adult plant stage. PLoS One, 2016;11.

[24] Chen X, Coram T, Huang X, Wang M, Dolezal A. Understanding Molecular mechanisms of durable and non-durable resitance to stripe rust in wheat using transcriptomics approach. Curr Genomics. 2013;14:111-126

[25] Jiang J, Zhao J, Duan W et al. TaAMT2;3a, a wheat AMT2-type ammonium transporter, facilitates the infection of stripe rust fungus on wheat. BMC Plant Biology. 2019;19. 
[26] Xu Q, Tang C, Wang X, et al. An effector protein of the wheat stripe rust fungus targets chloroplasts and suppresses chloroplast function. Nature Communications, 2019;1027.

[27] Garnica D, Upadhyaya N, Dodds P, Rathjen J. Strategies for wheat stripe rust pathogenicity identified by transcriptome sequencing. Plos One. 2013;8. 